\title{
ON THE STRONG RADICAL OF CERTAIN BANACH ALGEBRAS
}

\author{
by BERTRAM YOOD* \\ (Received 2nd September 1976)
}

\section{Introduction}

Let $A$ be a complex Banach algebra. By an ideal in $A$ we mean a two-sided ideal unless otherwise specified. As in (7, p. 59) by the strong radical $S$ of $A$ we mean the intersection of the modular maximal ideals of $A$ (if there are no such ideals we set $\mathcal{S}=A$ ). Our aim is to discuss the nature of $\mathfrak{S}$ and the relation of $\mathfrak{S}$ to $A$ for a special class of Banach algebras. Henceforth $A$ will denote a semi-simple modular annihilator Banach algebra (one for which the left (right) annihilator of each modular maximal right (left) ideal is not (0)). For the theory of such algebras see (2) and (9).

Our first aim is to describe $\subseteq$ in analytic terms. It turns out that $\subseteq$ is the annihilator in $A$ of the set of $x \in A$ for which the operation $L_{x}$ of left multiplication is a compact linear operator on $A$.

A more special class of Banach algebras is that of semi-simple Banach algebras with dense socle. That such an algebra $A$ need not be an annihilator algebra is seen by an example in (1). For such $A$ it is shown that $A / \mathbb{S}$ is a semi-simple annihilator algebra and that $\boldsymbol{A}$ is an annihilator algebra if $\mathcal{S}$ is an annihilator algebra.

\section{Preliminaries}

An idempotent $e$ in $A$ is called minimal if $e A(A e)$ is a minimal right (left) ideal. For a subset $W$ of $A$ let $L(W)(R(W)$ ) denote the left (right) annihilator of $W$ in $A$. Let $K$ be an ideal in $A$. By $\left(4\right.$, p. 162) $L(K)=R(K)$. We set $K^{a}=L(K)=R(K)$ and note that $K^{a a a}=K^{a}$. As a special case of (10, Lemma 5.1) a minimal idempotent $e$ of $A$ is in one of the ideals $K$ and $K^{a}$. As a consequence the socle $S_{A}$ of $A$ is a subset of $K \oplus K^{a}$. Also, see (9, Lemma 3.10), the minimal idempotents of $K$ are the minimal idempotents of $A$ which lie in $K$.

Let $M$ be a modular maximal ideal of $A$. Then $M^{a} \neq(0)$ so that $A=M \oplus M^{a}$. By (2, Theorem 6.4), $A / M$ is finite-dimensional. This could also be deduced from results in (8). Hence $M^{a}$ is a finite-dimensional simple Banach algebra. Note that $M=M^{a a}$. For otherwise $M^{a a}=A$ and $M^{a}=M^{a a a}=(0)$. Let $\mathfrak{M}$ denote the set of all modular maximal ideals of $A$. If $M_{1} \neq M_{2}$ in $\mathfrak{D}$, then $M_{1}^{a} \neq M_{2}^{a}$. As these are simple algebras, $M_{1}^{a} M_{2}^{a}=$ $M_{2}^{a} M_{1}^{a}=(0)$. Let $Q$ denote the closure of the algebraic sum of the $M^{a}, M \in \mathfrak{P}$. Then

$$
Q^{a}=\cap\left\{M^{a a}: M \in \mathfrak{D}\right\}=\Im \text {. }
$$

\footnotetext{
*This research was supported by the National Science Foundation, U.S.A.
} 
In view of $\left(7\right.$, p. 106), $Q$ is an annihilator algebra. Also, since $Q^{a}=Q^{a a a}$, we see that $\mathfrak{S}=\mathfrak{S}^{a a}$. This, in part, motivates our interest in ideals $K$ such that $K=K^{u a}$.

We say that a semi-simple Banach algebra $B$ is a left annihilator algebra if $L(I) \neq(0)$ for all closed right ideals $I \neq B$.

Proposition 2.1 Let $K$ be a closed ideal in the semi-simple Banach algebra $B$ where $K=K^{a a}$. Suppose that $B / K$ is a semi-simple left annihilator algebra and that $K$ is a left annihilator algebra. Then $B$ is a left annihilator algebra.

Proof. Let $I$ be a closed right ideal of $B, I \neq B$. We consider first the case where $I$ does not contain $K$. Then $K \cap I \neq K$. Hence there exists $w \in K, w \neq 0$, such that $w(K \cap I)=(0)$. Then $w I K=(0)$. Since $K$ is semi-simple $w I=(0)$. Therefore $L(I) \neq(0)$ in $B$.

Now we examine the case where $I=K$. As $K=K^{a u}$ then $K^{a} \neq(0)$ so that $L(I) \neq(0)$. Finally suppose that $I \supset K$ and $I \neq K$. Then $I / K$ is a closed right ideal of $B / K . I / K \neq(0)$ and $I / K \neq B / K$. By hypothesis there is $w \notin K$ such that

$$
(w+K)(I+K)=0+K \text {. }
$$

Therefore $w I \subset K$ so that $K^{a} w \subset L(I)$. We see readily, that $K^{a} w \neq(0)$ for otherwise $w \in K^{a a}=K$.

Proposition 2.2. Let e be a minimal idempotent in a semi-simple Banach algebra $B$. Then $e B$ is finite-dimensional if and only if $B e$ is finite-dimensional.

Proof. $B e B$ is a minimal (two-sided) ideal of $B$ so that its closure is a minimal closed ideal of $B$. Following Bonsall and Goldie (5, Theorem 13) we consider, corresponding to each $e y \in e B$, a bounded linear functional $f_{e y} \in(B e)^{*}$ defined by the equation

$$
f_{e y}(x e) e=\text { eyxe }
$$

for all $x e \in B e$. The mapping $G(e y)=f_{e y}$ is shown there to be a continuous one-to-one linear mapping of $e B$ into $(B e)^{*}$. Therefore $e B$ is finite-dimensional if $B e$ is finitedimensional.

We say that $e$ is a finite-dimensional minimal idempotent in the situation of Proposition 2.2.

\section{On modular annihilator algebras}

We investigate the strong radical $\subseteq$ of a semi-simple modular annihilator algebra A.

Lemma 3.1. If $p$ is a minimal idempotent of $Q$ then $p$ lies in some $M^{a}, M \in \mathscr{M}$ and $p$ is finite-diminsional idempotent.

Proof. Let $M \in \mathfrak{W}$. By the Gelfand-Mazur theorem, either $p M^{4} p=$ $\{\lambda p: \lambda$ complex $\}$ or $p M^{a} p=(0)$. In the former case $p \in M^{a}$. Suppose that $p$ lies in no 
$M^{a}, M \in \mathfrak{M}$. Then $\left(p M^{a}\right)^{2}=(0)$ so that $p M^{a}=(0)$ for each $M \in \mathfrak{M}$. Then $p Q=(0)$ which is impossible. Hence $p$ lies in some $M^{a}, p A \subset M^{a}$ and therefore $p$ is a finite-dimensional minimal idempotent.

Lemma 3.2. If $e$ is a finite-dimensional minimal idempotent of $A$ then $e \in Q$.

Proof. First $W=A e A$ is a simple Banach algebra. Consider the ideal $P^{e}$ of (2, Theorem 6.5). By that result $P^{e} \in \mathfrak{M}$. Moreover $P^{e} \subset A(1-e)$ so that $e \notin P^{e}$. Then $P^{e} \subset W^{a}$ since $W \cap P^{e}=(0)$. Therefore $P^{e} \oplus W=A$ while $e \in W$.

We consider the operators $L_{x}$ and $R_{x}$ on $A$ defined by $L_{x}(y)=x y$ and $R_{x}(y)=y x$. Let

$$
\begin{aligned}
& N_{L}=\left\{x \in A: L_{X} \quad \text { is a compact operator }\right\} \\
& N_{R}=\left\{x \in A: R_{x} \text { is a compact operator }\right\}
\end{aligned}
$$

Theorem 3.3. For a semi-simple modular annihilator algebra $A, \subseteq=N_{L}^{u}=N_{R}^{a}$.

Proof. If $e$ is a finite-dimensional minimal idempotent then, for any $x \in A, L_{e x}$ has finite-dimensional range and so is compact. Consider the collection $Z$ of finite sums of elements of this form ex. Via Lemmas 3.1 and 3.2 we see that $Z$ is the socle of $Q$. But $Q$ is an annihilator algebra so that $Z$ is dense in $Q$. Since $L_{z}$ is compact for each $z \in Z$ then $L_{x}$ is compact for all $x \in Q$. Hence $Q \subset N_{L}$.

Consider $x \in N_{L}, x \neq 0$. By the results of $(9, \S 3)$ every right ideal $\neq(0)$ in $A$ contains a minimal idempotent of $A$. Suppose $x \subseteq \neq(0)$. Then $x \subseteq$ contains a minimal idempotent $e$ where, perforce, $e \in \mathcal{S}$. On the other hand $N_{l}$ is an ideal in $A$ so that $L_{e}$ is a compact operator. It follows from the Riesz-Schauder theory that $\boldsymbol{e A}$ is finitedimensional. By Lemma 3.2, $e \in Q$ which is a contradiction. Therefore $x \widetilde{S}=(0)$. Consequently $x \in \widetilde{\varsigma}^{u}=Q^{a u}$.

We now have $Q \subset N_{L} \subset Q^{a a}$. Therefore

$$
\subseteq=Q^{a} \supset N_{L}^{a} \supset Q^{a a a}=Q^{a} \text {. }
$$

\section{On semi-simple Banach algebras with dense socle}

Henceforth let $A$ be a semi-simple Banach algebra with dense socle. $A$ is a modular annihilator algebra by $(9$, Lemma 3.11$)$. Let $K$ be an ideal in $A$. As noted in Section 2, the socle of $A$ is contained in $K \oplus K^{a}$. Hence $K \oplus K^{u}$ is dense in $A$. In particular $Q \oplus \Im$ is dense in $A$.

Theorem 4.1. A/S is a semi-simple annihilator Banach algebra.

Proof. Let $\pi$ be the natural homomorphism of $A$ onto $A / S$ and let $J$ be the radical of $A / \subseteq$. Now $V=\pi^{-1}(J) \cap Q$ is an ideal of $Q$ which is an annihilator algebra. Suppose $V \neq(0)$. Then $V$ contains a minimal idempotent $p$ of $Q$. As $\pi$ is one-to-one on $Q, \pi(p)$ is a non-zero idempotent in $J$. This is impossible and therefore $V=(0)$. Hence $\pi^{-1}(J) Q=(0)$ so that $\pi^{-1}(J) \subset Q^{a}=\Im$. Therefore $J=(0)$.

Let $I$ be a closed right ideal of $A / \Im, I \neq A / \Im$. Note that $\pi^{-1}(I)$ fails to contain $Q$ 
(for $\pi(Q)$ is dense in $A / S$ ). Then there exists $w \in Q$ such that $w \neq 0$ and $w \pi^{-1}(I) Q=$ (0). Obviously, $w \pi^{-1}(I) \subset Q$. As $Q$ is semi-simple we get $w \pi^{-1}(I)=(0)$ so that $\pi(w) I=(0)$ where $\pi(w) \neq 0$.

Proposition 4.2. If $\widetilde{S}$ is a left (right) annihilator algebra so is $A$.

Proof. We see this in two easy ways. First as $Q \oplus \subseteq$ is dense in $A$ and $Q$ is an annihilator algebra this follows from (7, p. 106). The result is also an immediate consequence of Theorem 4.1 and Proposition 2.1.

Theorem 4.3. The following statements are equivalent

(1) $\widetilde{S}=(0)$

(2) $A=N_{L}$

(3) $A=N_{R}$

Proof. Since $A$ has a dense socle, $N_{L} \oplus \widetilde{S}$ and $N_{R} \oplus \mathscr{S}$ are dense in $A$ by Theorem 3.3. Moreover $N_{L}$ and $N_{R}$ are closed ideals in $A$. For a related result see (2, Theorem 7.1).

Let $B_{1}$ be a semi-simple Banach algebra which is a dense ideal in another semi-simple Banach algebra $B_{2}$. The embedding is automatically continuous by (3, Proposition 2.2). We say that $B_{1}$ is an abstract Segal algebra in $B_{2}$. There is an extension literature on this notion of which we cite only (6).

A closed ideal $K$ in a semi-simple Banach algebra with dense socle need not have dense socle. We see no reason to expect $\mathbb{S}$ to have dense socle. Any $K$ and in particular $\mathbb{S}$ can be described as an abstract Segal algebra in a well-behaved Banach algebra.

Theorem 4.4. Let $K$ be a closed ideal in $A$. Then $K$ is an abstract Segal algebra in a semi-simple Banach algebra $B$ with dense socle. If $A$ is an annihilator algebra we can choose $B$ to be an annihilator algebra.

Proof. We know that $K \oplus K^{a}$ is dense in $A$. We take $B=A / K^{a}$ and let $\pi$ be the natural homomorphism of $A$ onto $A / K^{a}$. The mapping $\pi$ is one-to-one on $K$ and embeds $K$ as a dense ideal in $B$. The arguments of Theorem 4.1 show that $B$ is semi-simple. If $e$ is a minimal idempotent of $A, e \notin K^{a}$ then $\pi(e)$ is a minimal idempotent of $B$. Consequently $\pi\left(S_{A}\right)$ is contained in the socle of $B$ and so $B$ has dense socle.

Suppose that $A$ is an annihilator algebra. Let $I$ be a closed right ideal of $B . I \neq B$. $\pi^{-1}(I)$ is a right ideal of $A$ not dense in $A$. There exists $w \in A, w \neq 0$ where $w \pi^{-1}(I)=(0)$. Inasmuch as $\pi^{-1}(I) \supset K^{a}, w \in K^{a a}$. Since $K^{a} \cap K^{a a}=(0)$ we see that $\pi(w) \neq 0$ and $\pi(w) I=(0)$.

We say that an algebra is a strong radical algebra if it has no modular maximal ideal. $\mathfrak{S}$ is, as an algebra, a strong radical algebra.

Corollary 4.5. $S$ is an abstract Segal algebra in a semi-simple strong radical algebra $B$ with dense socle. 
Proof. Let $B$ be the algebra associated with $\mathfrak{S}$ as in the proof of Theorem 4.4 . Suppose that $B$ had a modular maximal ideal $M$. Since $\mathcal{S}$ is dense in $B, \mathcal{S} \not \subset M$. But then $\mathcal{S} \cap M$ is, by (7, Theorem 2.6.6), a modular maximal ideal of $\mathfrak{S}$. This is impossible. Therefore $B$ is a strong radical algebra.

The following remarks were kindly supplied by the referee. Theorems $3.3,4.1$ and 4.3 suggest various questions for a modular annihilator algebra $A$ such as when is $N_{L}$ or $N_{R}$ equal to $\mathfrak{S}^{a}$ ? If $N_{L}=N_{R}$, is their common value $\mathfrak{S}^{a}$ ? When is $A / \widetilde{S}$ strongly semi-simple? Theorem 4.3 supplies a partial answer to the first question, and would of course yield more if the third could be answered. The answer to the second in this general form is certainly negative.

For let $A=\mu_{a}^{x}$ where $a \in c_{0} \mid l_{1}$ (in the notation of (11)). Then $A$ is a Banach algebra with pointwise multiplication, is an ideal of $c_{0}$ and so is modular annihilator but does not have dense socle and so is not an annihilator algebra.

$A$ is a commutative $A^{*}$-algebra and therefore semi-simple and also strongly semi-simple. Thus if $N_{L}\left(=N_{R}\right)$ were equal to $\mathcal{S}^{a}$ we would have $A$ a completely continuous (c.c.) algebra. However a commutative $A^{*}$-algebra is an annihilator algebra if and only if it is a c.c. algebra. Thus for this $A, A \neq \widetilde{S}^{a}$. This example shows that Theorem 4.3 cannot be extended to modular annihilator algebras.

\section{REFERENCES}

(1) F. E. ALEXANDER, Some counter-examples of annihilator, dual and complemented $A^{*}$-algebras, J. London Math. Soc. (2) 8 (1974), 735-740.

(2) B. A. BARNES, Modular annihilator algebras, Canad. J. Math. 18 (1966), 566-578.

(3) B. A. BARNES, Banach algebras which are ideals in a Banach algebra, Pacific J. Math. 38 (1971), 1-7.

(4) F. F. Bonsall and J. DunCan, Complete Normed Algebras (Springer-Verlag, 1973).

(5) F. F. Bonsall and A. W. Goldie, Annihilator algebras, Proc. London Math. Soc. (3) 4 (1954), 154-167.

(6) J. T. Burnham, Closed ideals in subalgebras of Banach aigebras 1, Proc. Amer. Math. Soc. 32 (1972), 551-555.

(7) C. E. RICKART, General Theory of Banach Algebras (Van Nostrand, 1960).

(8) A. W. Tullo, Conditions on Banach algebras which imply finite-dimensionality, Proc. Edinburgh Math. Soc. 20 (1976), 1-5.

(9) B. YooD, Ideals in topological rings, Canad. J. Math. 16 (1964), $28-45$.

(10) B. YoOD, Closed prime ideals in topological rings, Proc. London Math. Soc. (3) 24 (1972), 307-323.

(11) D. J. H. Garling, On ideals of operators in Hilbert space, Proc. London Math. Soc. (3) 17 (1967), 115-138.

Pennsylvania State University

University Park, PA. 16802 U.S.A. 